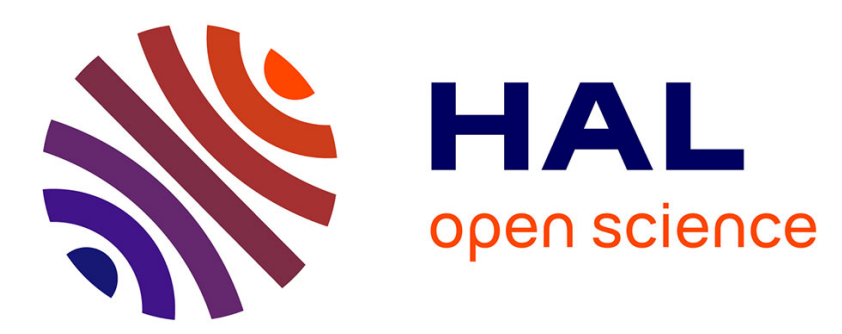

\title{
Numerical computations of rock dissolution and geomechanical effects for CO 2 geological storage
}

Kajetan Wojtacki, Jolanta Lewandowska, Philippe Gouze, Artur Lipkowski

\section{To cite this version:}

Kajetan Wojtacki, Jolanta Lewandowska, Philippe Gouze, Artur Lipkowski. Numerical computations of rock dissolution and geomechanical effects for CO 2 geological storage. International Journal for Numerical and Analytical Methods in Geomechanics, 2015, 39, pp.482-506. 10.1002/nag.2316 . hal01104204

\author{
HAL Id: hal-01104204 \\ https://hal.science/hal-01104204
}

Submitted on 19 Jan 2015

HAL is a multi-disciplinary open access archive for the deposit and dissemination of scientific research documents, whether they are published or not. The documents may come from teaching and research institutions in France or abroad, or from public or private research centers.
L'archive ouverte pluridisciplinaire HAL, est destinée au dépôt et à la diffusion de documents scientifiques de niveau recherche, publiés ou non, émanant des établissements d'enseignement et de recherche français ou étrangers, des laboratoires publics ou privés. 


\title{
Numerical computations of rock dissolution and geomechanical effects for $\mathrm{CO}_{2}$ geological storage
}

\author{
K. Wojtacki ${ }^{1 *}$, J. Lewandowska ${ }^{1, \dagger}$, Ph. Gouze ${ }^{2}$, A. Lipkowski ${ }^{3,4}$ \\ ${ }^{1}$ Laboratoire de Mécanique et Génie Civil, Université de Montpellier 2, Montpellier, France \\ ${ }^{\dagger}$ secondment to the French Ministry for Foreign Affaires and International Development, \\ Berlin, Germany \\ ${ }^{2}$ Géosciences, Université de Montpellier 2, CNRS, Montpellier, France \\ ${ }^{3}$ previously at Gdańsk University of Technology, Gdańsk, Poland \\ ${ }^{4}$ Gdańskie Inwestycje Komunalne, Gdańsk, Poland \\ *e-mail: kajetan.wojtacki@univ-montp2.fr
}

\begin{abstract}
The paper is motivated by the long term safety analysis of the $\mathrm{CO}_{2}$ geological storage. We present a methodology for the assessment of the geomechanical impact of progressive rock dissolution. The method is based on the use of X-ray tomography and the numerical dissolution technique. The influence of evolution of the microstructure on the macroscopic properties of the rock is analysed by using periodic homogenization method. The numerical computations show progressive degradation of all components of the stiffness (orthotropic) tensor. Moreover, the evolution of associated mass transfer properties (as tortuosity and conductivity tensors), by using the periodic homogenization method, is also calculated. The correlation between the mechanical parameters and the transfer properties during the dissolution process is presented. The results show that the highest increase of the hydraulic conductivity (in direction $Y$ ) is not associated with the highest decrease of Young modulus in this direction. Moreover, the highest decrease of Young modulus (in the direction $X$ ) is not associated with percolation in this direction. Finally, an incremental law to calculate settlement, in case of a rock with evolving microstructure, is proposed. The solution of the macroscopic
\end{abstract}


settlement problem under constant stress and drained conditions showed that the geomechanical effects of the rock dissolution are rather limited.

Key words: $\mathrm{CO}_{2}$ storage; homogenization; chemo-mechanical coupling; microstructure; X-ray tomography; numerical computations

\section{Introduction}

The geological storage of $\mathrm{CO}_{2}$ is intensively investigated as a solution to reduce emission of $\mathrm{CO}_{2}$ to the atmosphere. European Union climate and energy package [1] contains directives about carbon capture and storage (CCS). Its purpose is to create a legal framework for $\mathrm{CO}_{2}$ injection into deep geological formations and define procedures for a safe storage that minimizes the negative influence to the environment.

The CCS consists in capturing the $\mathrm{CO}_{2}$ at source and then its injection in the supercritical state into saline aquifer. During the geological storage, various mechanisms of trapping come into play [2]. In the short term after injection, migration of $\mathrm{CO}_{2}$ is blocked by the cap rock. With time, the complex physico-chemical processes occur. Chemical reactions may induce some important and irreversible changes of the rock properties (mechanical, hydrodynamical). Therefore, the long-term safety of the $\mathrm{CO}_{2}$ geological storage requires detailed understanding of the phenomena that have to be taken into account. Because of the complexity of natural systems, coupled effects of these processes are still not known completely.

The aim of the paper is to develop a macroscopic model of chemo-mechanical coupling related with the fourth stage of $\mathrm{CO}_{2}$ storage [3] when dissolution/precipitation are expected. The important objective is to find the link between the evolving microstructure of the material and the phenomena occurring at the microscopic scale, and the observed macroscopic behaviour. It will be possible as a result of application of the periodic homogenization method.

The conditions of the analysis will be representative of homogeneous dissolution taking place far from the injection well and at long time scale after injection. The chemical degradation of the material will be taken into account in a simplified way by performing the numerical erosion of the microstructure. The modifications of the microstructure trigger mechanical effects that will be considered in the framework of the incremental elasticity law. The proposed modelling is universal in the sense that it uses non-dimensional time scale that can be adjusted to a particular time dependent process. The paper is organized as follows. In section 2 the state of the 


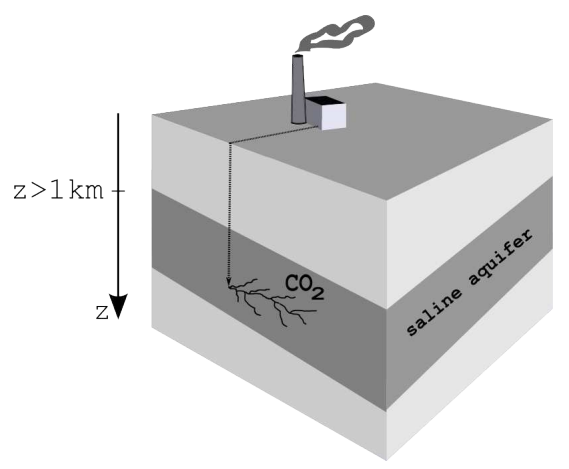

Figure 1: $\mathrm{CO}_{2}$ injection process.

art is presented. The macroscopic problem is presented in part 3. Section 4 contains short theoretical introduction of the numerical computations of elastic parameters based on the periodic homogenization. Next part describes preparation of samples on which computations were performed. In section 6 the results of computations of the degradation of the orthotropic elasticity tensor, together with analysis and interpretation, are presented. In this part the solution of the macroscopic problem of subsidence due to the rock degradation is also shown. Section 7 contains the analysis of the chemical degradation with respect to the transfer properties (tortuosity, conductivity) of the rock material. The correlations between mechanical and transfer properties are also provided. Finally, the last part contains conclusions.

\section{State of the art}

The chemo-mechanical coupling problem has been intensively studied in recent years. Chemical dissolution is the main phenomenon which increases porosity of rocks and affects their mechanical and physical properties.

The available publications on the chemo-mechanical coupling can be divided into two categories:

- experimental studies

- theoretical modelling and/or computational issues.

In the studies the authors distinguish between two types of conditions (related to the distance from the injection well): 
- near field conditions

- far field conditions.

Further, different studies focus on either short or long time behaviour.

Historically, first publications dealing with of chemo-mechanical coupling concerned concrete, mortar or cement paste. There is also a wide range of recently published papers, concerning experiments of chemical degradation of these materials tested in different conditions (see for example Nguyen et al. [4], Yurtdas et al. [5], Huang et al. 6]). The results obtained confirm a general weakening of the material during chemical degradation (loss of strength and stiffness). In the literature there are a lot of computational models describing chemo-mechanical coupling in concrete, mortar or cement paste due to different environmental conditions. Significant part of numerical models focus on leaching of calcium from cementitious structures. For example, a model based on scalar continuum damage was presented by Le Bellego et al. [7]. Ulm et al. 8] introduced an analytical model linked to the theory of Biot-Coussy. On the other hand, discrete model is shown in the paper of Chatzigeorgiou et al. [9]. Finally, homogenization approach was applied by Nguyen et al. [10] and Stora et al. [11].

The experimental studies considering $\mathrm{CO}_{2}$ injection into the rock reservoirs performed in the laboratory conditions, relatively close to the injection point, show different behaviours depending on physical parameters of injected fluid (temperature, pressure), chemical composition of injected fluid and initial rock parameters (see Luquot et al. [12], Gouze et al. [13], Eggermann et al. [14], Egermann et al. 2006 [15], Izgec et al. 2008 [16] among others). For example, Egermann et al. [14] performed an experimental study of chemical degradation of hydrocarbon rock reservoirs (which are considered by them as potentially the best candidates for $\mathrm{CO}_{2}$ geological storage) in the far field region. They analysed three different scenarios of dissolution: uniform, compact and wormholing. Xie et al. [17] performed hydrostatic and triaxial compression tests on samples of limestone, chemically degraded by dissolved $\mathrm{CO}_{2}$. The significant influence of chemical degradation in immersion condition on the mechanical behaviour of material, has been shown. They also showed that chemical degradation intensifies deformation of material with time. The $\mathrm{CO}_{2}$ injection to non-carbonate rock sample has been studied by Canal et al. [18]. They focused on the siliceous sandstone reservoirs. Their results suggest that mineral dissolution and cracking processes act in a synergetic way during the acidic injection. At the same time, they emphasize that increase of permeability noticed after a long time can be explained mainly by dissolution process of a solid phase. 
On the other hand some investigations showed the decrease of permeability at the beginning of injection of fluid into rock samples (Qajar et al. [19], Izgec et al. [16]). The authors relate this phenomenon with closing of pores throats by solid particles disconnected from the solid skeleton near to the inlet of injection. Later, increase and stabilization of permeability were noticed.

In the experimental research of behaviour of rock at long time scale after $\mathrm{CO}_{2}$ injection (Bemer et al. [20], Nguyen et al. [21]) it is assumed that porous aquifer is saturated by saline water and concentration of the dissolved $\mathrm{CO}_{2}$ is uniformly distributed in the pore space. The authors showed that chemical degradation can be considered as homogeneous at the sample scale. Moreover, their results show that porosity increases uniformly in time over the whole length of the sample. Because of increasing porosity material weakening occurs. Decrease of both, stiffness and shear strength, and increase of permeability have been also noticed. The experiments of permeability of cap rocks have been carried out by Bachaud et al. 22. Diffusion and permeability coefficients have been measured under reservoir thermodynamic conditions and compared before and after the alternation. Despite of increase of porosity, the initial low-value transport parameters were conserved. This was called by the authors "a non-catastrophic weakening of the material". Moreover, for few samples a decrease of diffusion coefficients was noticed.

The modelling of chemo-mechanical coupling is a subject of a large number of papers published in the recent years. The proposed approaches are very different. Several models were obtained by periodic homogenization method [23]-[27]. For example, Peter [23],24], Peter and Böhm [25] proposed a homogenisation analysis of elasticity problem with time evolving microstructure. In this model the equations are transformed to a fixed periodic reference geometry. This operation was necessary because during the evolution of the geometry, the periodicity was not conserved. The obtained model seems to be difficult to perform numerical simulations. In Lewandowska [26] a macroscopic incremental model of chemo-mechanical coupling by using the periodic homogenization was proposed. In this study it was assumed that the chemical dissolution of solid at the microscopic scale is governed by diffusion equation. Coussy [28] proposed a phenomenological model in which variations of poroelastic parameters are described as a function of irreversible porosity caused by chemical degradation. Numerical and computational aspects of chemo-mechanical modelling related to flow and transport phenomena during the injection process are presented in the papers by Doughty et al. [29], Kumar et al. [30], Hovorka et al. [31, analytical models of the same phenomena are presented by Saripalli et al. [32] and Nordbotten et al. 33. 
In conclusion, the state of the art shows that although there is a lot of publications dealing with the problem of chemo-mechanical coupling, the appropriate model of anisotropic rock dissolution with geomechanical effects in the context of $\mathrm{CO}_{2}$ geological storage, is still lacking. The difficulty resides in the fact that by its nature the problem is site specific in the sense the chemical composition of both the rock and saline water determine the behaviour of the system. The experiments are difficult (or even impossible) to perform because of the involved time-scale (hundreds or thousands of years). Moreover, the rock samples are difficult to obtain because of large costs of deep drilling. Additionally, the X-ray tomography can be taken with sufficient accuracy on small size samples only. For all these reasons, a universal and relatively simple model which would be able to link the macroscopic behaviour with the microscopic scale (evolving microstructure), is still searched.

\section{Formulation of the problem}

\subsection{Description of the problem}

Let us consider a saturated volume of rock located deeply in the underground, shown in Figure 1. We assume that all upper layers apply constant stress $\sigma_{0}$ on the aquifer and that the variations of stress caused by human or environment activities are negligible, comparing to the weight of all upper layers. We consider the so called 'far field' and/or long term situation which means that the $\mathrm{CO}_{2}$ was completely dissolved in the saline aquifer. The presence of $\mathrm{CO}_{2}$ in water creates the acid environment, therefore, dissolution of carbonates of rock material begins. The progressive dissolution causes changes of porosity, and variations of material properties, this process can take thousands of years. In the analysis we assume that the porous rock is homogeneous and periodic. Since, in general, the rock material is non-periodic we will have to periodize it by using the assumption of orthotrophy (see section 5.2. This assumption implies that periodic cell has got three planes of symmetry and the axes of anisotropy correspond to the natural anisotropy of the rock. The objective is to formulate an incremental model, in the framework of linear theory of elasticity, that will be able to capture the subsidence due to the chemical degradation of solid under a constant load. 


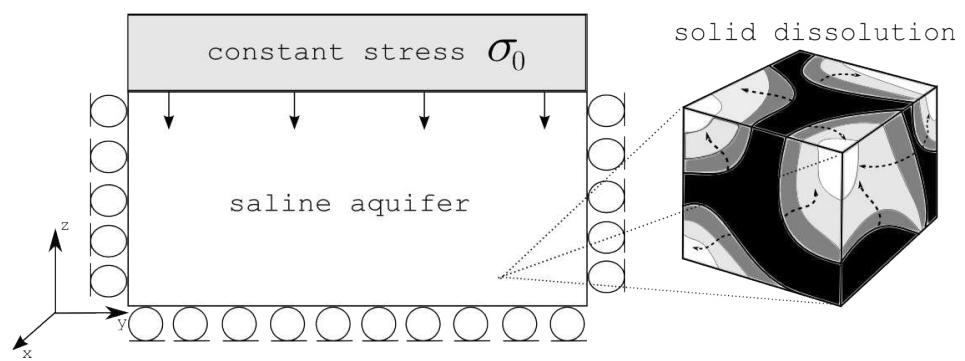

Figure 2: Macroscopic rock dissolution problem with evolving microstructure.

\subsection{Formulation of the macroscopic incremental law of elas- ticity}

Let us consider a macroscopic, one dimensional problem of settlement (creep) taking place in a rock medium characterized by three dimensional microstructure presented in Figure2, where axis $Z$ is parallel to the gravity axis and position of all axes remain unchanged during the process of settlement (no rotation during one-dimensional deformation in the direction $Z$ ). We assume that saline water with no resistance can flow out from the aquifer (drained conditions) and that the external stress is constant in time. In order to derive an incremental law of elasticity, let us assume the validity of Hooke's law in which material properties (e.g. Young modulus) and deformation depend on porosity $\varphi$

$$
\sigma_{0}=E(\varphi) \epsilon(\varphi)
$$

By calculating the derivative with respect to porosity we obtain

$$
0=\frac{\mathrm{d} E(\varphi)}{\mathrm{d} \varphi} \epsilon(\varphi)+E(\varphi) \frac{\mathrm{d} \epsilon(\varphi)}{\mathrm{d} \varphi} .
$$

Equation 2 can be presented in the following form

$$
0=\epsilon(\varphi) \mathrm{d} E(\varphi)+E(\varphi) \mathrm{d} \epsilon(\varphi),
$$

which is equivalent to

$$
0=\epsilon(\varphi)[E(\varphi+\mathrm{d} \varphi)-E(\varphi)]+E(\varphi)[\epsilon(\varphi+\mathrm{d} \varphi)-\epsilon(\varphi)] .
$$

After an algebraic transformation we can write

$$
\epsilon(\varphi+\mathrm{d} \varphi)=\epsilon(\varphi)\left[2-\frac{E(\varphi+\mathrm{d} \varphi)}{E(\varphi)}\right]
$$




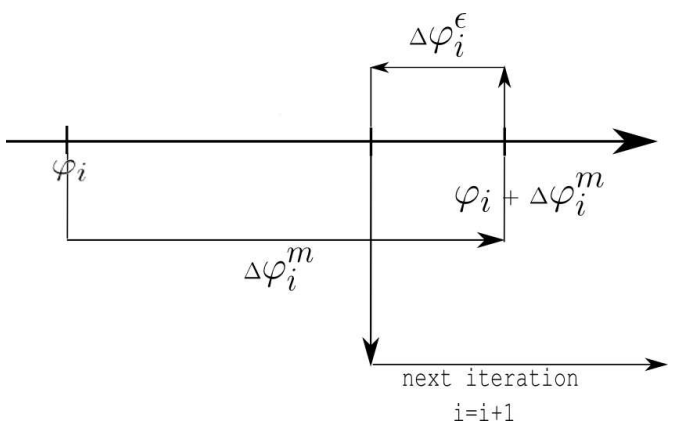

Figure 3: Illustration of algorithm.

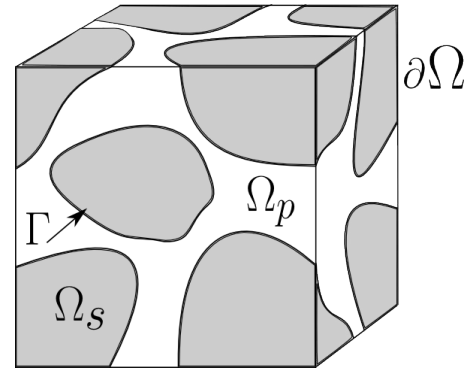

Figure 4: The period $\Omega$.

By introduction of an iterative notation we obtain

$$
\epsilon_{i+1}=\epsilon_{i}\left[2-\frac{E_{i+1}}{E_{i}}\right] .
$$

We must notice that our problem is a feedback process. At each step there is a change of porosity due to mass loss (dissolution). This process causes the decrease of Young modulus and increase of deformation. At the same time, each increment of mechanical deformation (settlement) leads to decrease of porosity and to associated increase of Young modulus. For clarity we illustrated it in Figure 3 , where $\varphi_{i}$ is the current value of porosity of $i$ step, $\Delta \varphi_{i}^{m}$ is the change of porosity caused by loss of mass and $\Delta \varphi_{i}^{\epsilon}$ is the variation of porosity caused by deformation. The next step $(i+1)$ starts from value of porosity $\varphi_{i}+\Delta \varphi_{i}^{m}-\Delta \varphi_{i}^{\epsilon}$. If we assume that the skeleton material is incompressible with respect to the porous material, than the macroscopic deformation can be considered as equal to the change of porosity. Finally, we write

$$
\Delta \varphi_{i}^{\epsilon}=\frac{\sigma_{0}}{E\left(\varphi_{i}+\Delta \varphi_{i}^{m}\right)}
$$

where $E\left(\varphi_{i}+\Delta \varphi_{i}^{m}\right)$ means the value of Young modulus for porosity $\left(\varphi_{i}+\Delta \varphi_{i}^{m}\right)$, which is taken from the degradation function. Finally, to solve the problem we need to know the degradation functions of material parameters. The procedures of obtaining the macroscopic values of material parameters based on the periodic homogenization are presented in section 4. 


\section{Theoretical background of numerical computa- tions of elasticity tensor}

\subsection{Poroelasticity}

In this subsection, the result of homogenization process for poroelasticity problem [34] [36] is presented. The linear elasticity problem of the solid skeleton is represented by the period, illustrated in Figure 4, where is the solid boundary, is the solid volume and is the pores volume. The solid material is characterized by microscopic fourth-order stiffness tensor $a_{i j k h}$. After the homogenization process, the macroscopic problem is formulated by a set of differential equations. The equilibrium equations become

$$
\frac{\partial}{\partial x_{i}}<\sigma_{i j}^{0}>=0 \quad \text { in } \quad \Omega_{s}
$$

where $\sigma_{i j}^{0}$ is the zero order macroscopic stress tensor and the symbol $\langle\cdot\rangle$ is the volume average in the sense

$$
<\cdot>=\frac{1}{\Omega} \int_{\Omega_{s}} \cdot d \Omega .
$$

The constitutive law becomes

$$
<\sigma_{i j}^{0}>=C_{i j k h} e_{x k h}\left(\vec{u}^{(0)}\right)
$$

where $e_{x k h}$ is the macroscopic strain tensor and $\vec{u}^{(0)}$ is the zero order macroscopic displacement vector. The macroscopic fourth order stiffness tensor $\mathbf{C}$ is defined as

$$
C_{i j k h}=<a_{i j k h}+a_{i j l m} e_{y l m}\left(\vec{\xi}^{k h}\right)>
$$

where $a_{i j k h}$ is the microscopic fourth order stifness tensor and $\vec{\xi}^{k h}(\vec{y})$ is a local displacement periodic vector as a particular solution of the following problems

$$
\frac{\partial}{\partial y_{i}}\left[a_{i j l m} e_{y l m}\left(\vec{\xi}^{k h}\right)+a_{i j k h}\right]=0 \quad \text { in } \quad \Omega_{s}
$$

and

$$
\left[a_{i j l m} e_{y l m}\left(\vec{\xi}^{k h}\right)+a_{i j k h}\right] n_{i}=0 \quad \text { on } \quad \Gamma,
$$

where $n_{i}$ is a component of the unit normal external vector with respect to $\Omega_{s}$. If we know the microstructure (period), then we are able to calculate the full stiffness 
tensor. In order to solve the problem we have to find $\vec{\xi}^{k h}(\vec{y})$ [34], 36], 37], which corresponds to the unit macroscopic strain tensor $\mathbf{E}^{k h}=\left(e_{k} \otimes e_{h}+e_{h} \otimes e_{k}\right) / 2$. Where symbol $\otimes$ means the tensorial product. To obtain full stiffness tensor in the anisotropic case, the local displacement vector field $\vec{\xi}^{k h}(\vec{y})$ has to be found by performing the following elementary tests

1. three traction tests, where $h=k$ and $h, k \in(1,2,3)$

$$
\mathbf{E}^{11}=\left(\begin{array}{ccc}
1 & 0 & 0 \\
0 & 0 & 0 \\
0 & 0 & 0
\end{array}\right), \quad \mathbf{E}^{22}=\left(\begin{array}{ccc}
0 & 0 & 0 \\
0 & 1 & 0 \\
0 & 0 & 0
\end{array}\right), \quad \mathbf{E}^{33}=\left(\begin{array}{ccc}
0 & 0 & 0 \\
0 & 0 & 0 \\
0 & 0 & 1
\end{array}\right)
$$

2. three shearing tests where $h \neq k$ and $h, k \in(1,2,3)$

$$
\mathbf{E}^{12}=\left(\begin{array}{ccc}
0 & 1 / 2 & 0 \\
1 / 2 & 0 & 0 \\
0 & 0 & 0
\end{array}\right), \quad \mathbf{E}^{23}=\left(\begin{array}{ccc}
0 & 0 & 0 \\
0 & 0 & 1 / 2 \\
0 & 1 / 2 & 0
\end{array}\right), \quad \mathbf{E}^{13}=\left(\begin{array}{ccc}
0 & 0 & 1 / 2 \\
0 & 0 & 0 \\
1 / 2 & 0 & 0
\end{array}\right)
$$

\subsection{Symmetric boundary conditions for poroelasticity prob- lem}

When the period presents three planes of symmetry, the periodicity conditions can be replaced by the symmetry conditions imposed on the external boundary $\partial \Omega$, so the local boundary value problem can be solved in the $1 / 8$ of the whole periodic cell. The type of the symmetry boundary conditions depends on the mechanical test that is being performed 38 ]

- for traction test

$$
\begin{array}{lll}
\vec{u}_{N}=\overrightarrow{0}, & \text { on } & \partial \Omega \\
\vec{\sigma}_{T}=\overrightarrow{0} & \text { on } & \partial \Omega
\end{array}
$$

- for shearing test in the plane $(\vec{i}, \vec{j})$

- on the external planes orthogonal to the axis $\vec{i}$ and $\vec{j}$

$$
\begin{aligned}
\vec{u}_{T} & =\overrightarrow{0} \\
\vec{\sigma}_{N} & =\overrightarrow{0}
\end{aligned}
$$


- on the external planes orthogonal to the axis $\vec{k}$

$$
\begin{aligned}
& \vec{u}_{N}=\overrightarrow{0} \\
& \vec{\sigma}_{T}=\overrightarrow{0}
\end{aligned}
$$

where $\vec{u}_{N}, \vec{u}_{T}$ are displacements in normal and tangential directions respectively. Analogically $\vec{\sigma}_{N}$ and $\vec{\sigma}_{T}$ represent normal and tangential stress vectors. The set $(\vec{i}, \vec{j}, \vec{k})$ represents unit vectors of the standard basis. In order to compute the full macroscopic stiffness tensor $\mathbf{C}$ (equation 11) the local boundary value problem (equations 12, 13) for a particular microstructure has to be solved six times.

\section{Microstructure, X-ray tomography and numer- ical dissolution}

\subsection{X-ray tomography}

In the paper the geometry of a natural rock material is investigated. It is a sample of an oolitic limestone from Mondeville formation of the Middle Jurasic age (Paris Basin) [13]. The chemical composition of the rock corresponds to a magnesiumcalcite formula $\mathrm{Ca}_{\alpha} \mathrm{Mg}_{\beta} \mathrm{CO}_{3}$ with $\alpha=0.99$ and $\beta=0.01$. The digital image of the microstructure has been obtained by X-ray tomography. The size of the image is $1067 \times 1067 \times 2000$ pixels, where 1 pixel $=5.06 \mu \mathrm{m}$, so the physical dimensions are $5.4 \times 5.4 \times 10.12 \mathrm{~mm}^{3}$. The raw images were processed (see for example [39] ) in order to obtain a binary image displaying the solid fraction and the void fraction (or porosity). The initial porosity of the sample calculated by ImageJ software is $\phi=9.3 \%$.

\subsection{Sample preparation}

By using ImageJ application the image of the microstructure has been imported, as shown in Figure 5. In order to know the size of the REV for the particular material, the porosity as function of the dimension of the REV, taken from seven different regions in the sample, was analysed (Figure 6). Note that the porosity criterion

is only an approximation when we are interested in mechanical properties. From Figures 6 and 7 it can be clearly seen that all lines converge toward the average 


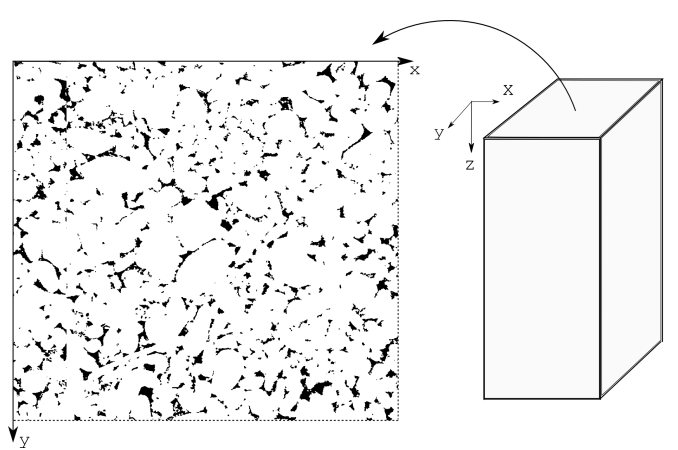

Figure 5: Upper view of the microstructure in $X Y$ plane of size $1067 \times 1067$ pixels. White part corresponds to the solid phase, black to pore space.

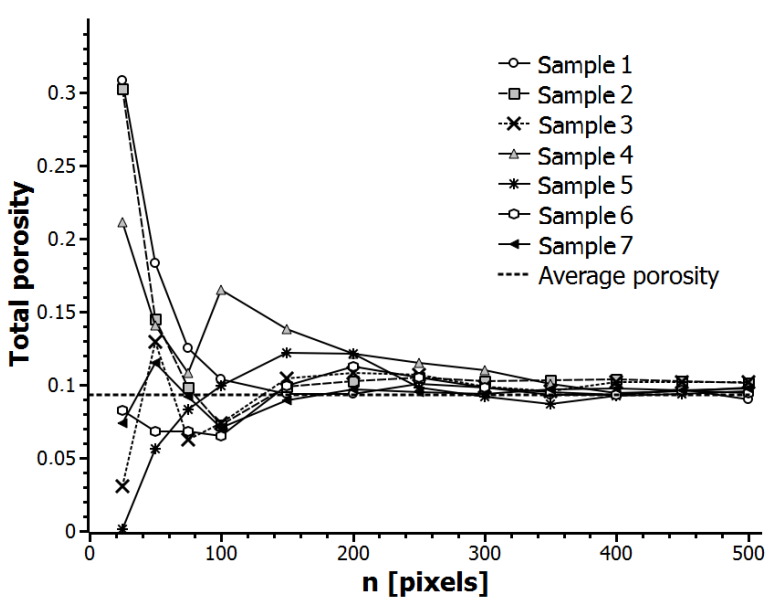

Figure 6: Porosity as a function of edge length of the REV $n$.

value of porosity $\varphi=9.3 \%$ when the dimension of the REV approaches 200 pixels. However, the microstructure of the size $200 \times 200 \times 200$ pixels appeared to be too large for numerical computations. Therefore, in what follows we adopted a particular strategy. We assumed that the rock microstructure is periodic, and that the period presents three reflectional planes of symmetry. Then, seven cubic sub-samples of the size $50 \times 50 \times 50$ pixels, each representing $1 / 8$ of the complete period, were selected for further analysis. So, the real size of the analysed microstructure was $100 \times 100 \times 100$ pixels. These samples are located in the central part of the microstructure as shown in Figure 7. The samples localization are the vertices of a symmetrical hexagon and the centre point. Each sample was further processed (see section 5.3). Finally, on seven cubic sub-samples computations were performed at different dissolution states and all results of numerical computations were interpreted together (see section 6.2 for further discussion).

In the numerical computations, each sample will be considered as a $1 / 8$ of the period. That means that if we want to reconstruct the period we have to perform the so called "periodization process" by using symmetries, as shown in Figure 8 . We can see three reflection symmetries by using three perpendicular planes. Axis $Z$ is parallel to the gravity axis and together with two other main axes $X$ and $Y$ do not rotate during the one dimensional settlement due to the dissolution process. At the first step we mirror the microstructure to the plane $Z X$, so we obtain $1 / 4$ of the period. Nextly, we do the same with respect to the plane $X Y$, and finally, 


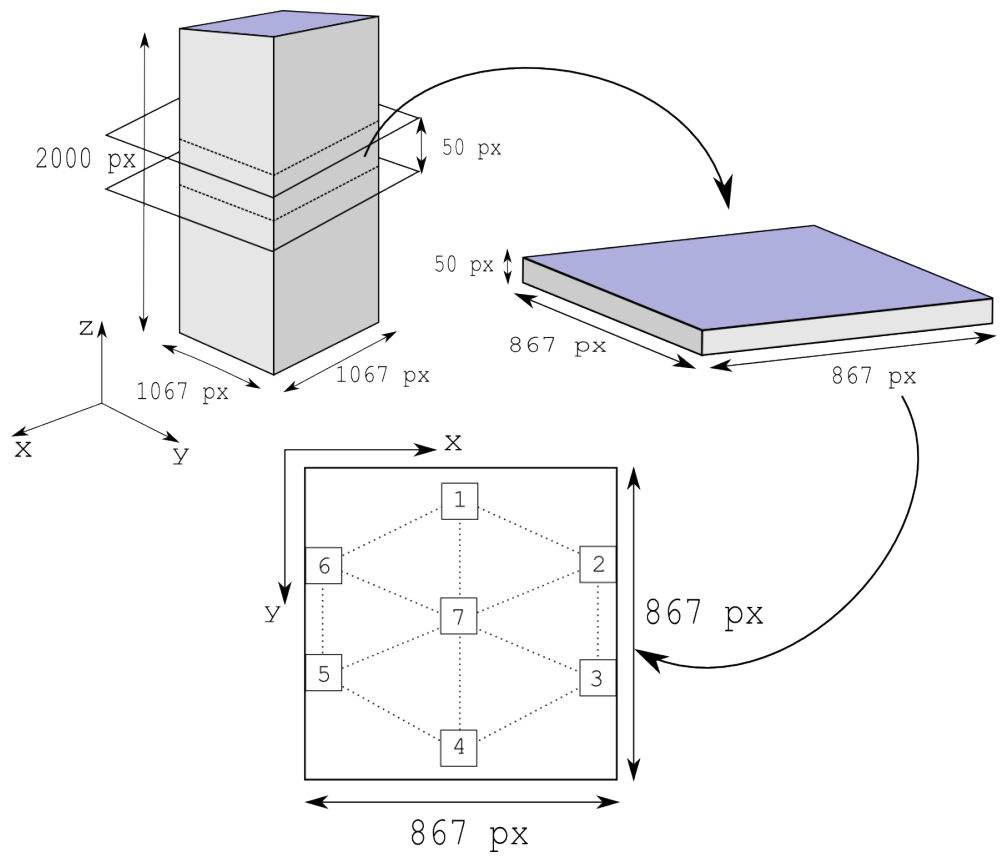

Figure 7: Samples preparation - cross section of the microstructure.

to get the periodic cell, we mirror with respect to the plane $Z Y$. Note that $X, Y, Z$ are assumed to be the axes of natural anisotropy.

\subsection{Chemical dissolution}

The aim is to perform the parametric study to present the dependence of mechanical properties in the whole range the theoretical porosity changes (in reality dissolution process can be limited). We assumed that dissolution of the porous matrix is the

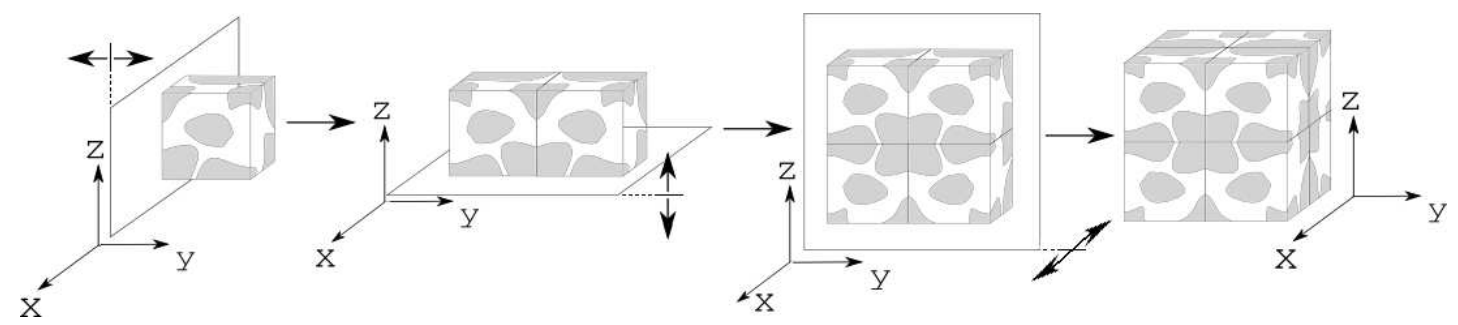

Figure 8: Periodization process. 


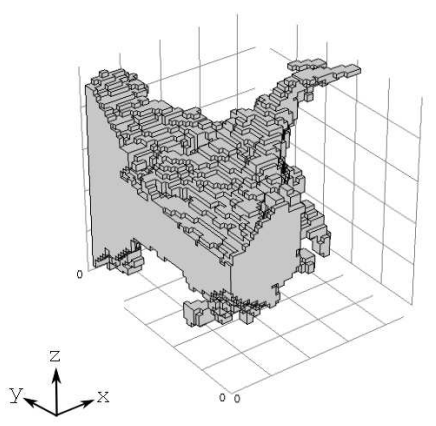

(a) Pores volume

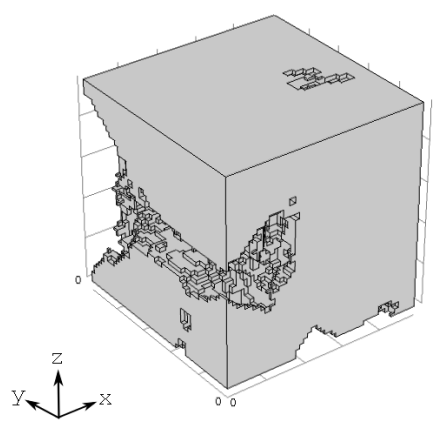

(b) Solid skeleton

Figure 9: Microstructure of sample 4 (1/8 of the periodic cell); $\varphi_{\text {total }}=16.3 \%$, $\varphi_{\text {effective }}=14.9 \%$.

only process responsible for chemo-mechanical coupling. The chemical degradation has been performed as a numerical dissolution of the solid phase by ImageJ. For each of seven selected samples the 'erode 3D' option for solid has been used. The main assumption is that the solid part of each microstructure dissolves uniformly in all directions. It consist in removing one pixel in all directions in single non-dimensional time step. This assumption was appropriate due to the uniformity of the chemical composition and long time scale (long term behaviour) in which the distribution of pressure and $\mathrm{CO}_{2}$ concentration in saline water is approximately uniform in the investigated aquifer $([20],[21])$. The numerical erosion of the microstructure is universal and offers various options. Erosion may be performed in a particular direction (directional dissolution), completely random or according to any other assumption.

For each of seven samples we performed the numerical dissolution. As an example in Figures 9-10, the dissolution process of sample 4 is shown. We can see the evolving pores and the solid skeleton volumes. The total porosity varies between $16.3 \%$ to $37.8 \%$. Note that $\varphi_{\text {effective }}$ corresponds to effective porosity of samples in which unconnected pores and unconnected solid parts have been deleted. In Figure 9 percolation in $Y$ and $Z$ direction appears. At the last state of dissolution the percolation in all directions exists, as shown in Figure 10. As to the solid part, it is connected in each direction at each solid state. 


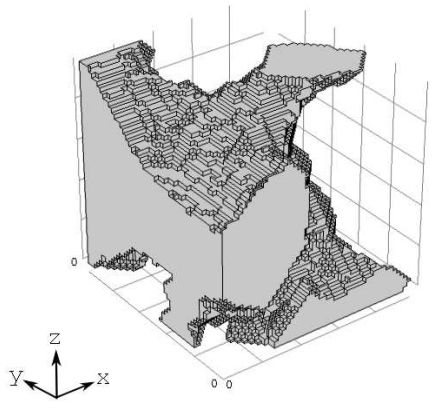

(a) Pores volume

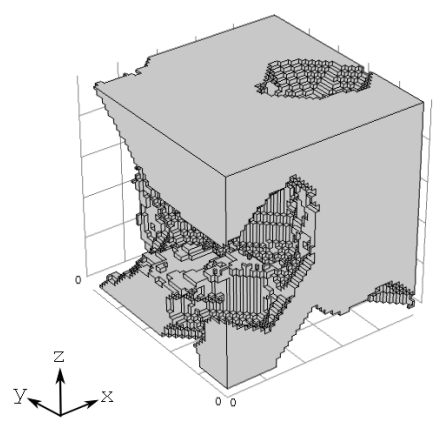

(b) Solid skeleton

Figure 10: Microstructure of sample 4 ( $1 / 8$ of the periodic cell); $\varphi_{\text {total }}=37.8 \%$, $\varphi_{\text {effective }}=37.8 \%$.

\section{Numerical computations of stiffness tensor and subsidence}

\subsection{Proposed methodology}

The methodology we proposed can take into account the geometry evolution effects of real anisotropic rock sample on mechanical properties and to estimate the subsidence due to chemical degradation. The problem of settlement, formulated in section 3.2 , is an iterative process for which continuous functions of material parameters dependent of porosity variations have to be known. To predict the deformation due to time dependent changes of rock microstructure we propose the general methodology presented in Figure 11. The first step is to prepare the digital image of the microstructure of the rock which will be investigated. The second step consists in preparation of samples to perform mechanical tests at different degraded states. The next step is to perform, for each state, appropriate numerical computations by solving local boundary value problem and calculating the full macroscopic stiffness tensor. Afterward, the data should be processed and the degradation functions have to be fitted. The last step is to use obtained functions to compute the subsidence. 


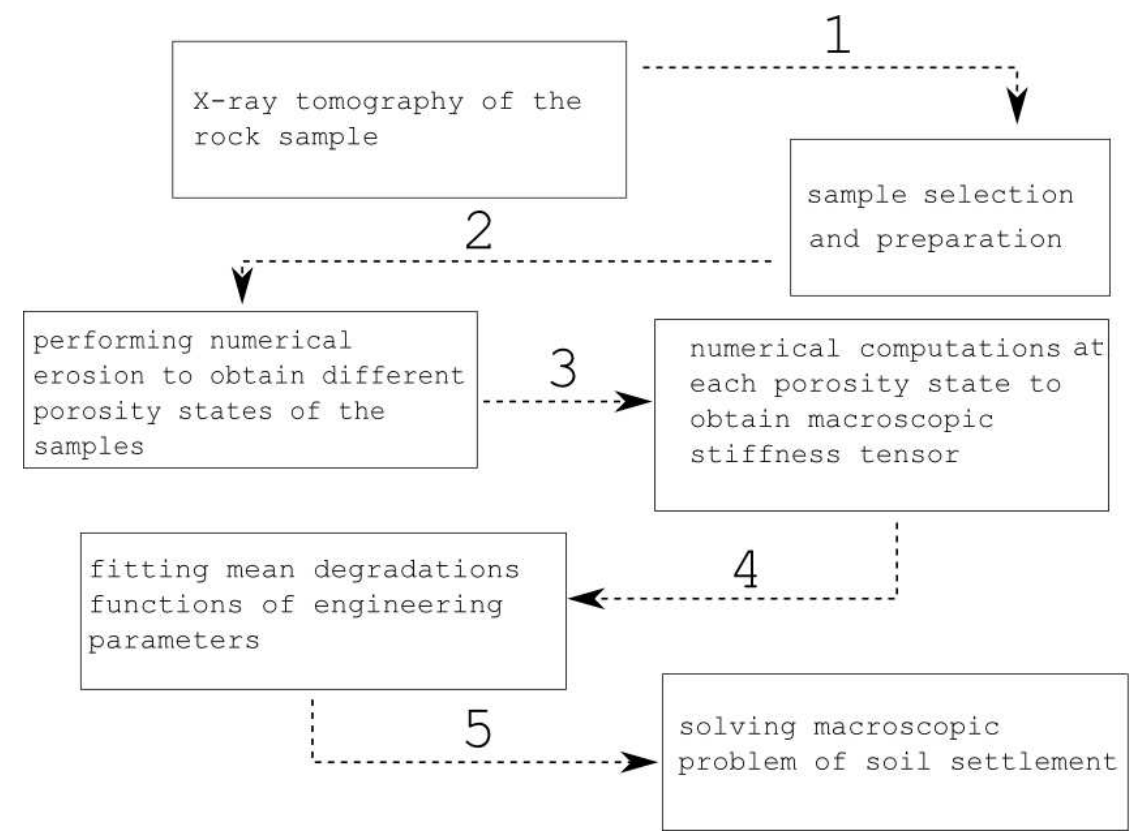

Figure 11: Algorithm of the proposed methodology.

\subsection{Computations of degradation functions of material pa- rameters}

In order to compute degradation functions of mechanical parameters the numerical computations using homogenization approach were performed. We adopted the following strategy. The computations were carried out for all seven samples at the initial state of the rock and three selected samples at different states of chemical degradation. Than, all results were interpreted together to define the degradation functions. The choice of such approach can be justified by the experimental results [20], [21]. Indeed, the experiments showed that the spatial variations of measured porosity within the rock material were of the same order that porosity variations of a sample due to the dissolution.

The computations were carried out for all seven samples at the initial state of the rock and three selected samples (sample 1, sample 4, sample 7) at different degraded states.

The numerical computations consisted in solving the local boundary value problem (eq. 12 and eq. 13) with symmetry boundary conditions (equation 14 or equation 15 and equation 16). We assumed that the solid material (at microscopic scale) 
is isotropic with following values of Young modulus and Poisson ratio: $E_{\text {micro }}=27.7$ $G P a, \nu_{\text {micro }}=0.27$. These values correspond to the average material parameters of Euville limestone from the basin of Paris, measured by Nguyen et al. [27].

In each case, equation 11 was used to obtain full macroscopic stiffness tensor.

The computations has been performed by using FEM code with a number of mesh elements varying from 100000 to 250000.

\subsection{Results}

All results (for initial and degraded samples) were interpreted together as functions of porosity. The mean degradation functions were fitted to all samples data. The character of variations for each parameter can be described by exponential decay function in the form $A \exp (-x / B)+C$, where constants $A, B, C$ were fitted by least square method. The correlation coefficient varies between 0.94 and 0.99 .

In Figures 12 - 20 the variation of engineering parameters $\left(E_{x}, E_{y}, E_{z}, G_{y z}, G_{z x}\right.$, $\left.G_{x y}, \nu_{x y}, \nu_{y z}, \nu_{z x}\right)$ with porosity are shown . Nine of them are independent, since material is orthotropic [40]. This property was systematically checked by veryfing the relations between Poisson ratios and Young moduli

$$
\frac{\nu_{i j}}{E_{i}}=\frac{\nu_{j i}}{E_{j}} \quad i \neq j \quad \text { and } \quad i, j \in(x, y, z)
$$

The relative error for each test was less than $0.1 \%$. Also the zero-elements of the matrix were confirmed by computations.

Figures 12, 14 show Young modulus for each direction as function of porosity. The drop of $E_{x}$ is the most significant. Figures 15 , 17 present three shear moduli. As we can see, the most significant decrease concerns $G_{z x}$. For Poisson ratios $\nu_{x y}, \nu_{y z}$, $\nu_{z x}$, no general trends appear (Figures 18-20). Finally, the analysis of the results presented in Figures 12,17. show that degradation data for sample 4 is very close to the plot of mean degradation function. For this reason sample 4 was chosen to further computations (see section 7).

The numerical results could be compared with experimental data. Unfortunately, no experiments on orthotropic material are available in the literature. The comparison with experimental results of degradation of isotropic material provided in ([20], 21]) shows a similar trend of dependence of mechanical parameters on porosity. 


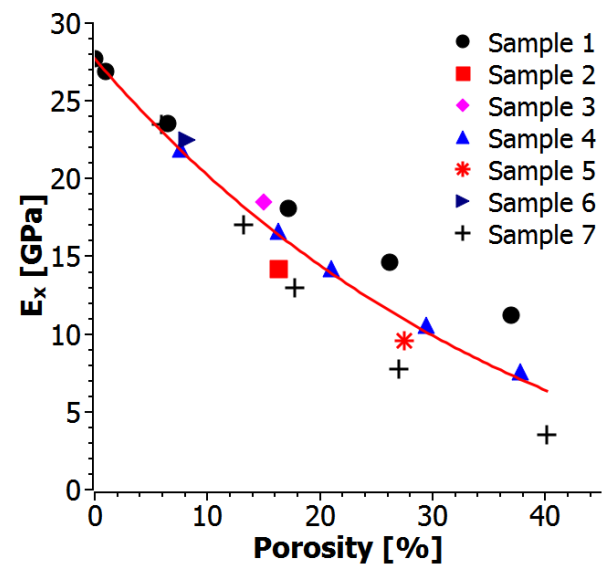

Figure 12: Degradation function of $E_{x}$.

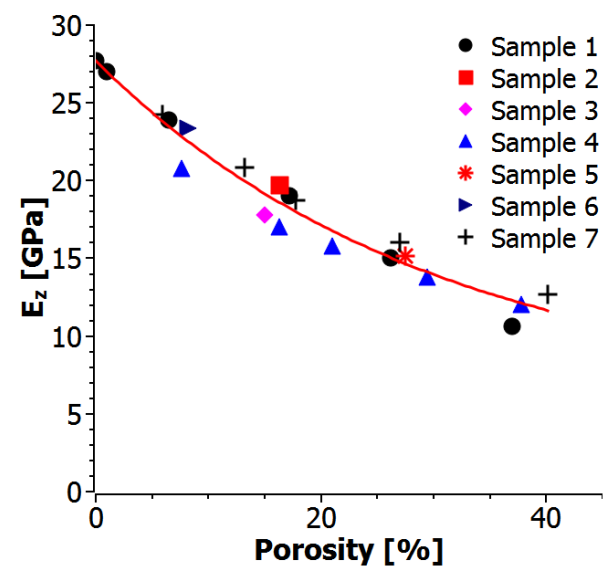

Figure 14: Degradation function of $E_{z}$.

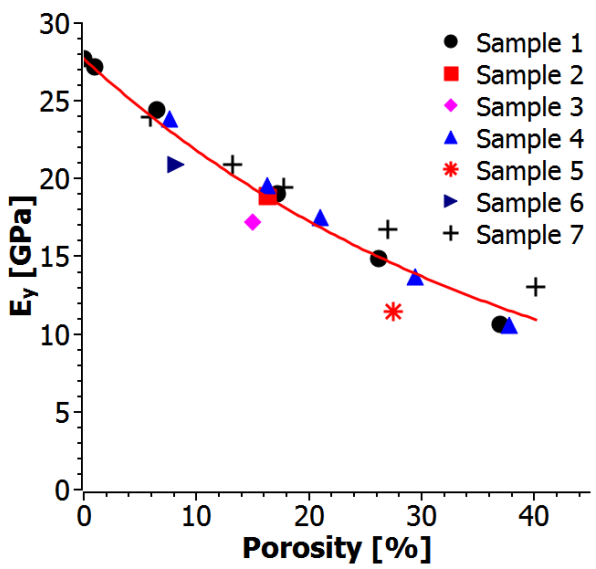

Figure 13: Degradation function of $E_{y}$.

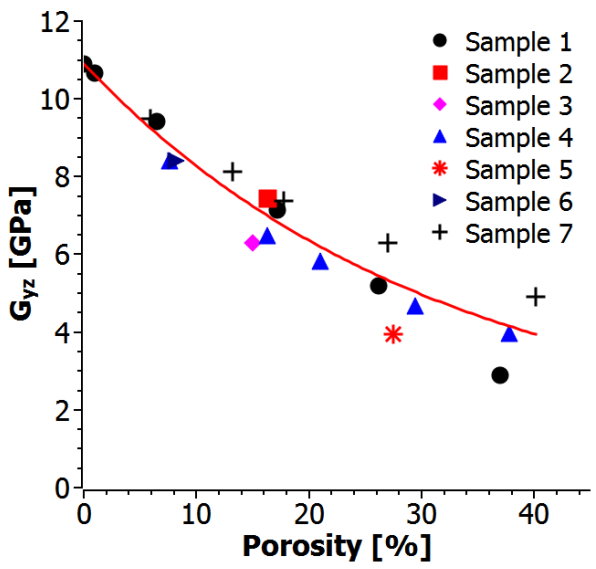

Figure 15: Degradation function of shear modulus $G_{y z}$. 


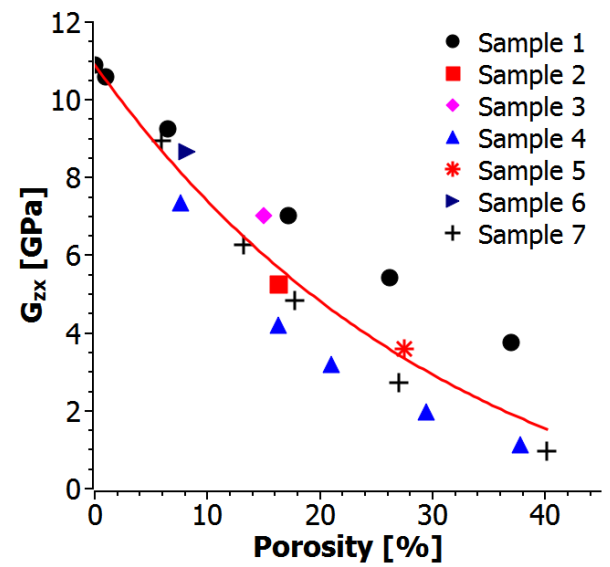

Figure 16: Degradation function of shear modulus $G_{z x}$.

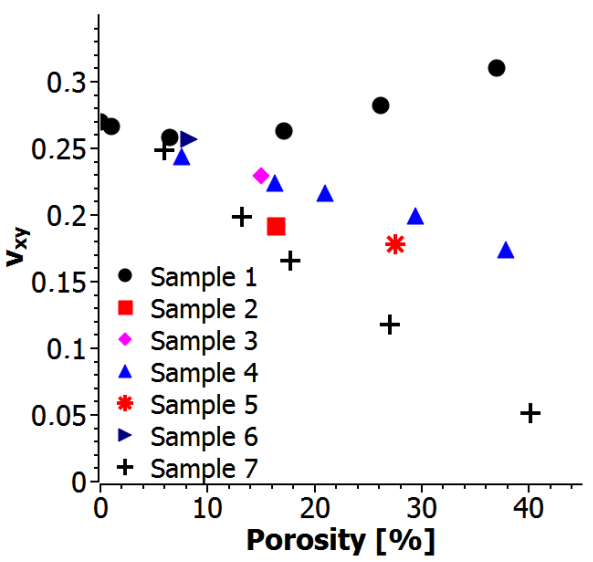

Figure 18: Poisson ratio $\nu_{x y}$ as a function of porosity.

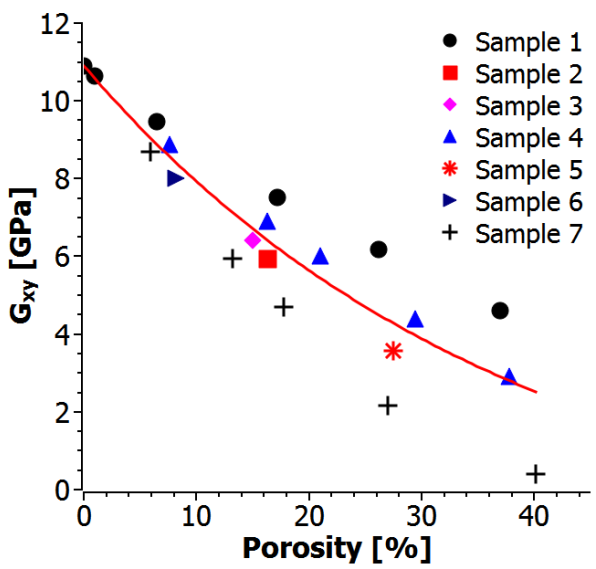

Figure 17: Degradation function of shear modulus $G_{x y}$.

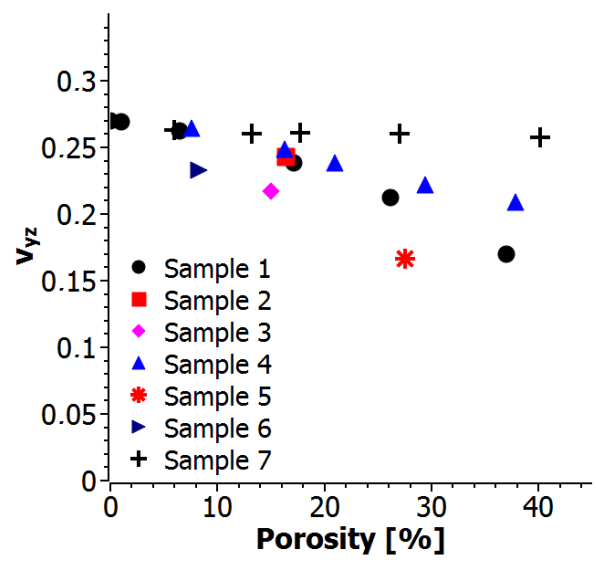

Figure 19: Poisson ratio $\nu_{y z}$ as a function of porosity. 


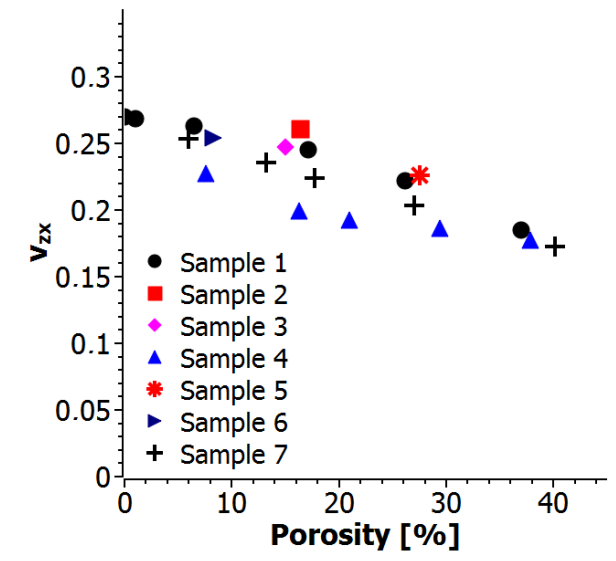

Figure 20: Poisson ratio $\nu_{z x}$ as a function of porosity.

\subsection{Solution of the macroscopic boundary problem}

The one-dimensional problem of subsidence defined in section 3.2 was solved by using the degradation function of the Young modulus in the $z$ direction (Figure 14), given by

$$
E_{z}=A \exp (-\varphi / B)+C
$$

where $A=16.78 \mathrm{GPa}, B=0.16, C=10.02 \mathrm{GPa}$ and $\varphi$ is a porosity. It is assumed that vertical stress is constant and equal $\sigma_{z}=20 \mathrm{MPa}$. This stress corresponds to the weight of layer of $1 \mathrm{~km}$ of the unit volumetric weight $20 \mathrm{kN} / \mathrm{m}^{3}$. In Figure 21 the strain variation $\epsilon_{z}=\Delta s / h$ (decreased by the value of initial deformation $\left.\epsilon_{0}=\sigma_{0} / E_{z}\left(\varphi_{0}\right)\right)$ as a function of porosity is shown. We observe the increase of strain to the value of $-0.65 \times 10^{-3}$, while the porosity varies from the 0.093 to 0.40 (initial and final values). The corresponding Young modulus varies from $E_{z}=20.19$ GPa to $E_{z}=12.19 \mathrm{GPa}$, respectively. It can be said, that in the framework of the assumption of long term behaviour, drained condition and uniform dissolution the macroscopic effects of subsidence are very limited. 


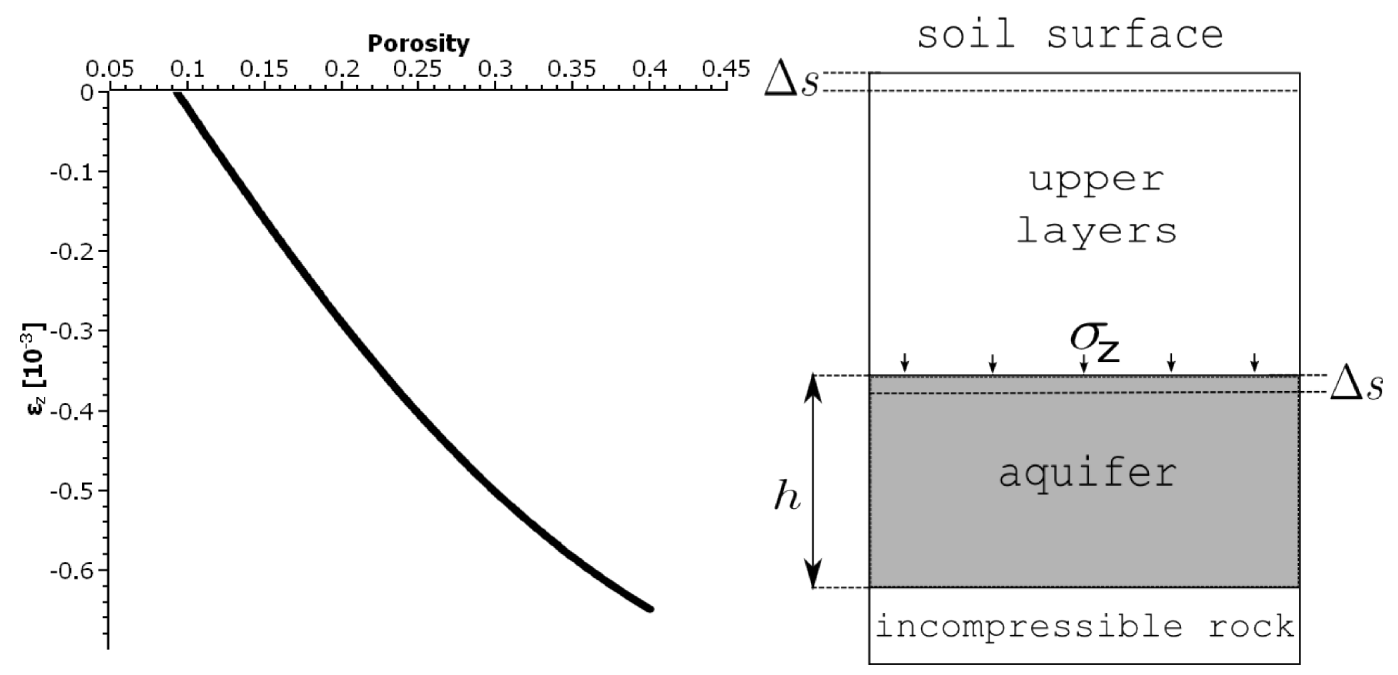

Figure 21: Settlement problem: vertical macroscopic deformation $\epsilon_{z}$.

\section{Mechanical parameters versus morphological prop- erties of the rock material}

The upscaling method by periodic homogenization provides the link between the microscopic and macroscopic scales. Therefore, the variations of mechanical parameters of the rock can be analysed in relation with the microstructure and its morphological properties. In this section the permeability, diffusion and tortuosity tensors for selected rock sample (sample nr 4) at the initial and degraded states will be calculated. In view of previous assumption of existence of three planes of symmetry, $X, Y, Z$ are the principal axes of these tensors. This situation does not change during the one-dimensional settlement due to the material degradation process. The aim is to analyse the correlation between those parameters and the mechanical properties when microstructure is subjected to evolution.

\subsection{Mechanical properties versus diffusive properties}

In order to calculate the effective diffusion tensor for a particular periodic microstructure the following local boundary value problem for the vector $\vec{\chi}$ has to be solved 
[41], [42]

$$
\begin{gathered}
\frac{\partial}{\partial y_{i}}\left[D\left(\mathbb{I}_{i j}+\frac{\partial \chi_{j}}{\partial y_{i}}\right)\right]=0 \quad \text { in } \quad \Omega_{f} \quad \text { and } \quad i, j \in(1,2,3), \\
N_{i}\left[D\left(\mathbb{I}_{i j}+\frac{\partial \chi_{j}}{\partial y_{i}}\right)\right]=0 \quad \text { on } \quad \Gamma \quad \text { and } \quad i, j \in(1,2,3),
\end{gathered}
$$

where $\vec{\chi}$ is y-periodic and its volumetric average is equal to zero. $D$ is the diffusion coefficient at the microscopic scale. $\vec{N}$ is the external normal unit vector to the $\Omega_{f}$ (pore domain). In the computations presented in this paper the periodicity conditions were replaced by the symmetry conditions as proposed in [43]. The effective diffusion tensor is given by [41], 42]

$$
D_{i j}^{e f f}=\frac{1}{\Omega} \int_{\Omega_{f}} D\left(\mathbb{I}_{i j}+\frac{\partial \chi_{j}}{\partial y_{i}}\right) d \Omega
$$

and the tortuosity tensor by [4]

$$
\tau_{i j}=\frac{1}{\Omega} \int_{\Omega_{f}}\left(\mathbb{I}_{i j}+\frac{\partial \chi_{j}}{\partial y_{i}}\right) d \Omega .
$$

Note that tortuosity is usually defined in phenomenological approaches, see for example [4], as the ratio

$$
\tau=\frac{\Lambda}{\Lambda^{\prime}},
$$

where $\Lambda$ is the straight distance between two positions of a particle, $\Lambda^{\prime}$ is length of trajectory connecting these two positions. According to this definition, tortuosity varies between 0 and 1. In Figure 22 the tortuosity of the sample 4 as a function of the increasing porosity, occurring during chemical degradation, is presented. We can observe the increase of the tortuosity in the direction $Y$ and $Z$, and constant (almost zero value) in the $X$ direction. In general, the increase of tortuosity is related to the decrease of the particles trajectory in the pores [44]. Therefore, it can be interpreted as "straightening" of pores (pores become less tortuous). In the $X$ direction there is no percolation until the effective porosity reaches about $38 \%$. Figure 23 presents tortuosity tensor components divided by the effective porosity. It can be said, that kinetics of changes of tortuosity in relation to increase of the effective porosity is different for $\tau_{z}$, than $\tau_{y}$. It can be seen that the rate of "straightening" of trajectories for $\tau_{y}$ is almost constant during dissolution.

In Figures 2629 relations between tortuosity tensor components and mechanical parameters are shown. For all parameters the increase of tortuosity $\left(\tau_{y}\right.$ and $\left.\tau_{z}\right)$ is associated with the decrease of Young and shear moduli. 


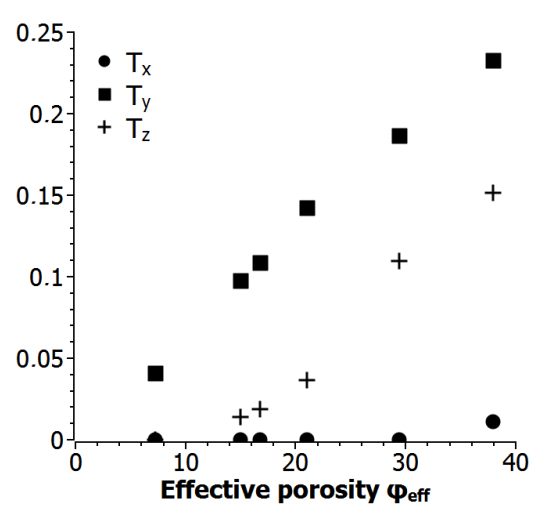

Figure 22: Tortuosity parameters for each direction as a function of effective porosity.

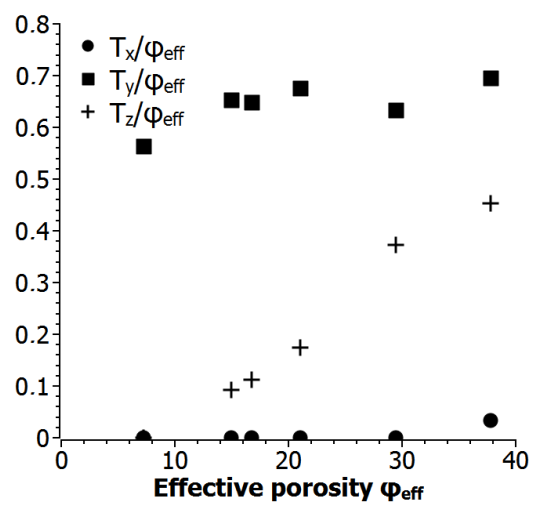

Figure 23: Tortuosity parameters for each direction divided by corresponding effective porosity.

Finally, the specific surface of the material as being equal to the pore surface divided by the volume of the microstructure for sample 4 (in evolution), was calculated. In Figure 33 the specific surface as a function of the effective porosity, is drawn. We can see a general trend of increase of specific surface with porosity. For porosity equal to $21 \%$ a local decrease appears, that can be explained by fusion of pores forced by chemical dissolution. Generally speaking, the increase of specific surface can explain the drop of all mechanical parameters (Figure 24 and Figure 25), since it means the increase of surface available for dissolution and loss of mass.

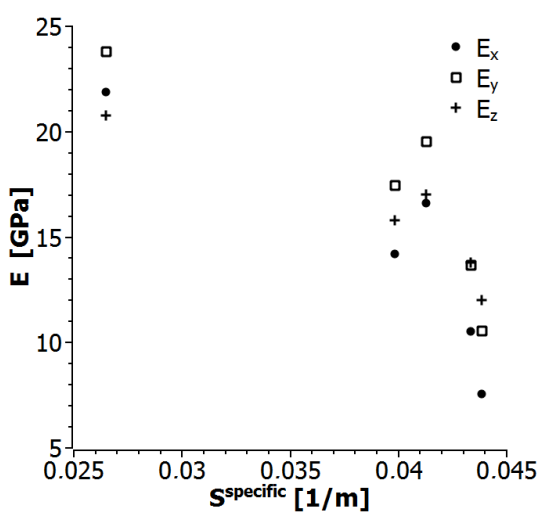

Figure 24: Young modulus as function of specific surface.

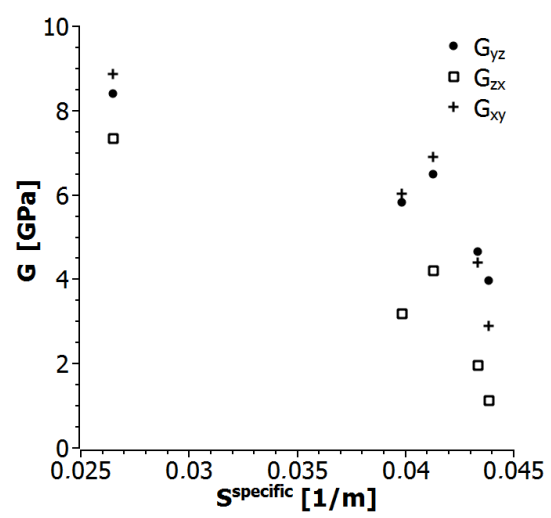

Figure 25: Shear modulus as function of specific surface. 


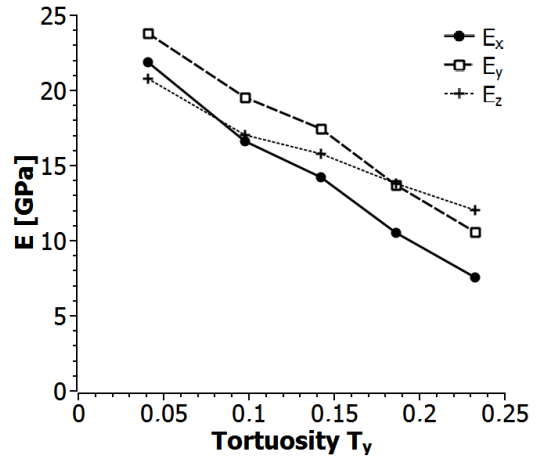

Figure 26: Young modulus as function of tortuosity $\tau_{y}$.

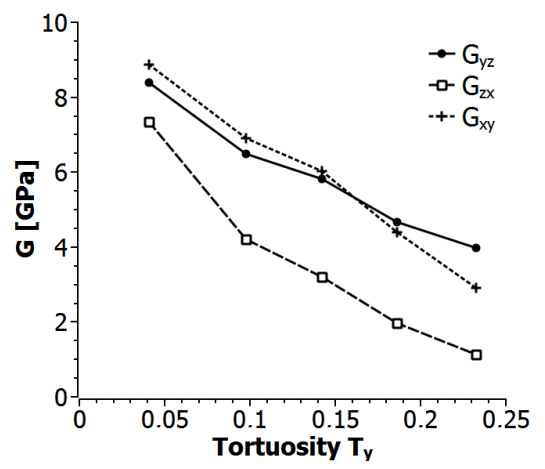

Figure 28: Shear modulus as function of tortuosity $\tau_{y}$.

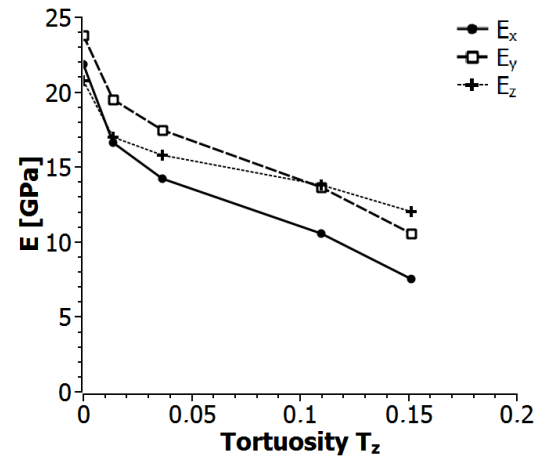

Figure 27: Young modulus as function of tortuosity $\tau_{z}$.

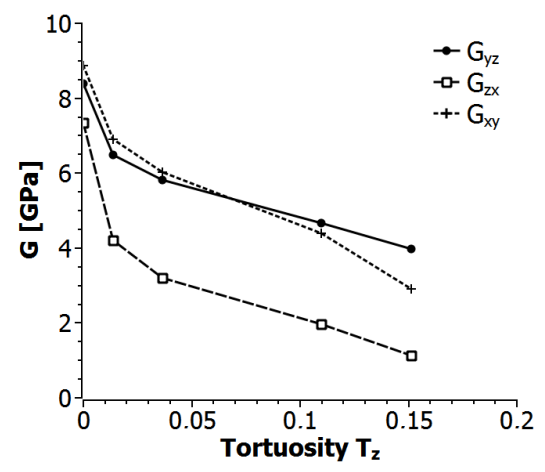

Figure 29: Shear modulus as function of tortuosity $\tau_{z}$.

\subsection{Mechanical properties versus permeability}

The permeability of porous rock can be calculated by solving a local boundary value problem of water flow in the pore domain. Since the microstructure presents symmetries, the problem to be solved in order to compute the permeability in the $Z$ direction (for example), is as follows

1. Stokes equation

$$
\eta \Delta \vec{v}-\nabla p=0 \quad \text { in } \quad \Omega_{f}
$$




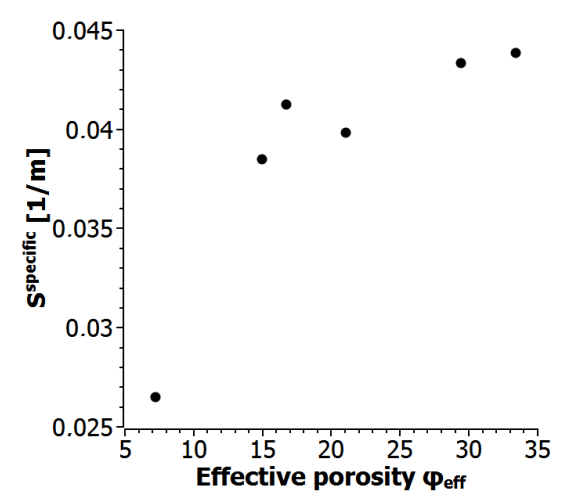

Figure 30: Specific surface as function of effective porosity.

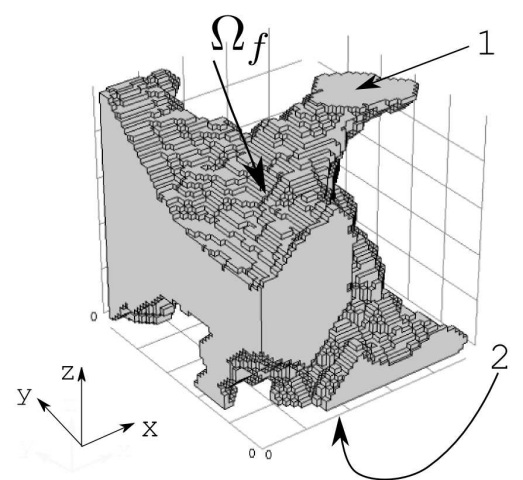

Figure 31: Pore volume of sample 4 in degraded state, with indicated notation for boundary conditions.

2. Incompressibility condition

$$
\nabla \vec{v}=0 \quad \text { in } \quad \Omega_{f}
$$

with following boundary conditions (Figure 31)

- on the surface 1: pressure $p_{1}=1$

- on the surface 2: pressure $p_{2}=0$

- on all other external surfaces: slip condition

- on all internal solid surfaces: non-slip condition

The permeability $K_{z}$ can be calculated from the Darcy law that is valid at the macroscopic scale [35]

$$
K_{z}=-\frac{<v_{z}>\eta}{\frac{\partial}{\partial z} p},
$$

where $\partial p / \partial z$ means macroscopic gradient of pressure in the $Z$ direction, $\left\langle v_{z}\right\rangle$ is the average fluid velocity in the $Z$ direction and $\eta$ is the dynamic viscosity. In the problem formulation we used the result of theoretical analysis, concerning the scale separation, for the case of symmetry of the period, as in [43]. The computations were performed for sample 4 in its initial and degraded states. The results of computations of three components $\left(K_{x}, K_{y}, K_{z}\right)$ as functions of the effective porosity are presented 


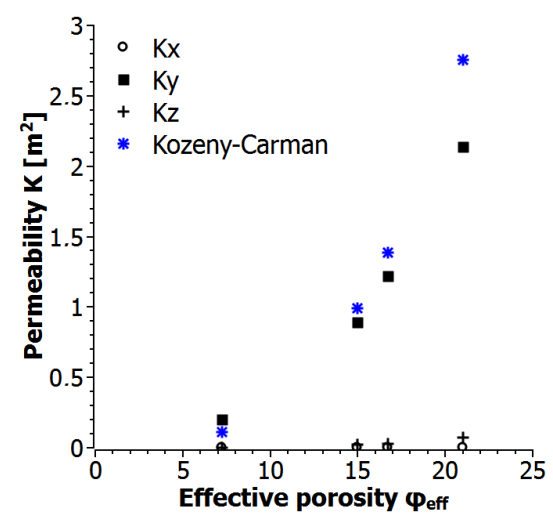

Figure 32: Comparison of permeability tensor elements and Kozeny-Carman permeability.

in Figure 32, We can observe a significant increase of $K_{y}$ while $K_{z}$ increases only slightly and $K_{x}$ remains zero (no percolation in $X$ direction in this range of porosity). In the same figure, the Kozeny-Carman classical solution for isotropic media is shown for comparison. Similar study was performed by Narsilio et al. [45].

In Figures 33,36 the relations between Young and shear moduli, and non-zero elements of permeability tensor, are presented. A general trend of decrease of the mechanical parameters as function of increasing permeability is confirmed.

\subsection{Conclusions}

The numerical computations confirmed that the loss of mass of solid (increase of porosity) is linked with weakening of the material. Although, porosity systematically increases, the hydraulic conductivity increases significantly only in the direction $Y$. On the other hand, the Young modulus in the direction $Y\left(E_{y}\right)$ is not the most affected by the degradation. It seems that in the direction $Y$ a preferential path appeared within which a lot of contacts in the directions $X$ and $Z$ had been lost. Since the most significant decrease of Young modulus occurred in the direction $X$, we can suppose that the preferential path cut the maximum number of contacts in the $X$ direction with respect to others $(Y$ and $Z)$. It is also confirmed by the decrease of two shearing moduli linked to the direction $X$, namely $\left(G_{x y}\right.$ and $\left.G_{z x}\right)$. The modulus $G_{x y}$ decreases less rapidly than $G_{z x}$ that can be explained by the fact that the number of lost contacts in the direction $Y$ is less than in the direction $Z$. Similarly, we can expect that the drop of modulus $G_{y z}$ is less than the two others 


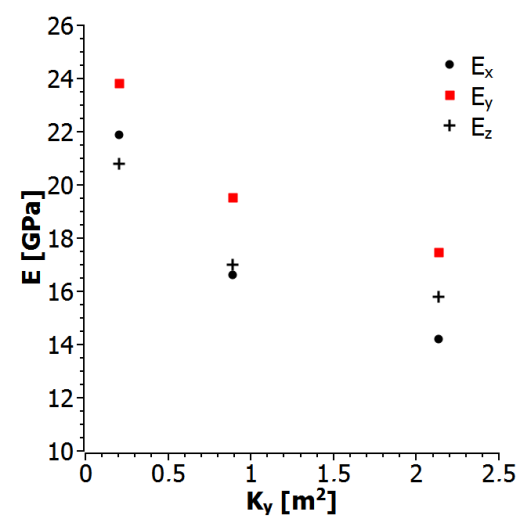

Figure 33: Young moduli as a function of $K_{y}$.

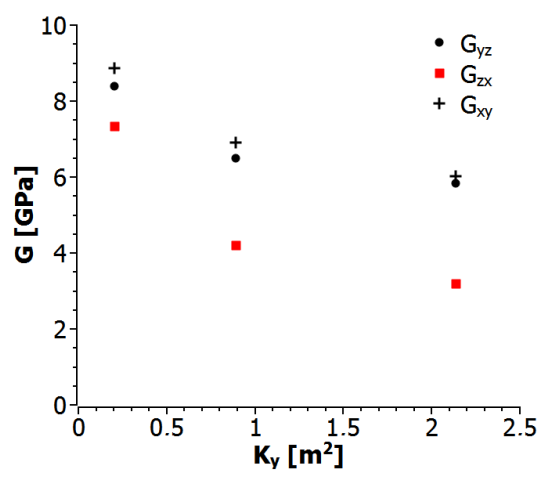

Figure 35: Shear moduli as a function of $K_{y}$.

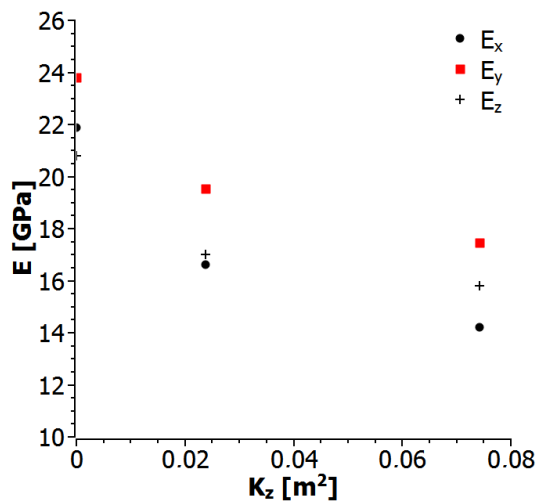

Figure 34: Young moduli as a function of $K_{z}$.

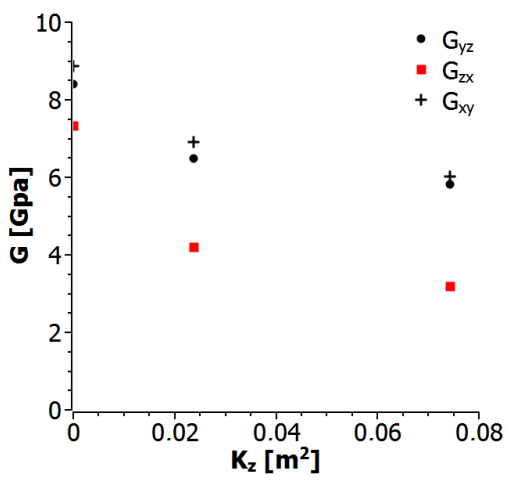

Figure 36: Shear moduli as a function of $K_{z}$. 
since the lost of contacts in the direction $Y$ and $Z$ is less important than in the direction $X$. The latter is confirmed in Figures $15,17$.

Interestingly, the highest decrease of Young modulus $E_{x}$ (and associated shearing moduli $G_{x y}$ and $G_{z x}$ ) is not related with the important variations of the transfer parameters (tortuosity, conductivity). Therefore, we can say more generally, that the decrease of mechanical parameters is not necessarily related with percolation.

As far as tortuosity is concerned, we observed that the rate of increase of the tortuosity is constant in the direction $Y$, but is subjected to significant changes in the direction $Z$. Nevertheless, the change of kinetics of tortuosity is not reflected in the kinetics of changes of mechanical parameters in the respective directions.

These conclusions have to be verified by performing more numerical computations and experiments.

\section{Final Conclusions}

A methodology linking dissolution of rock at the microscopic scale during the geological storage of $\mathrm{CO} 2$, and the geomechanical behaviour of the saline aquifer, is proposed. The performed numerical computations for an orthotropic microstructure showed progressive degradation of all components of the stiffness tensor that follow an exponential decay function.

The relation between the mechanical parameters and the transfer properties (tortuosity and conductivity tensors) during the dissolution process was also studied. It was found that the high increase of conductivity (in the direction $Y$ ) does not correspond (as could be expected) to the highest weakening of the Young modulus in this direction. Moreover, the highest decrease of Young modulus (in the direction $X)$ is not associated with percolation in this direction.

The results clearly show that the morphological information about the microstructure (contacts) can be very useful in the qualitative explanation of the chemical degradation process.

The solution of the macroscopic settlement problem under constant stress and drained conditions showed that the geomechanical effects of the rock dissolution are rather limited. 


\section{Ackonwledgement}

We would like to acknowledge the funding of the $\mathrm{PhD}$ to the first author from the French Ministry of Higher Education and Research.

\section{References}

[1] European Commission, Directive 2009/31/EC of the European Parliment and of the Council of 23 April 2009 on the geological storage of carbon dioxide and amending Council Directive 85/337/EEC, European Parliament and Council Directives 2000/60/EC, 2001/80/EC, 2004/35/EC, 2006/12/EC, 2008/1/EC and Regulation (EC) No. 1013/2006. Official Journal of the European Union 2009; $5(6): 114145$.

[2] Metz B, Davidson O, de Coninck H, Loos M, Meyer L. (eds.) IPCC Special Report on Carbon Dioxide Capture and Storage. Cambridge University Press: Cambridge, United Kingdom and New York, USA, 2005.

[3] Bachu S, Bonijoly D, Bradshaw J, Burruss R, Holloway S, Christensen N. P, Mathiassen O. M. $\mathrm{CO}_{2}$ storage capacity estimation: Methodology and gaps. International Journal of Greenhouse Gas Control, 2007; 1: 430-443.

[4] Nguyen V. H, Colina H, Boulay C, Torrenti J. M, Nedjar B. Chemo-mechanical coupling behaviour of leached concrete Part I: experimental results. Nuclear Engineering and Design, 2007; 237: 2083-2089.

[5] Yurtdas I, Xie S. Y, Burlion N, Shao J. F, Saint-Marc J, Garnier A. Influence of chemical degradation on mechanical behavior of a petroleum cement paste. Cement and Concrete Research, 2011; 41: 412-421.

[6] Huang B, Qian C. Experiment study of chemo-mechanical coupling behavior of leached concrete. Construction and Building Materials, 2011; 25: 2649-2654.

[7] Le Bellégo C, Pijaudier-Cabot G, Gérard B, Dubé J, Molez L. Coupled mechanical and chemical damage in calcium leached cementitious structures. Journal of Engineering Mechanics, 2003; 129: 333-341.

[8] Ulm F. J, Lemarchand E,Heukamp F. H. Elements of chemomechanics of calcium leaching of cement-based materials at different scales. Engineering Fracture Mechanics, 2003; 70: 871-889. 
[9] Chatzigeorgiou G, Picandet V, Khelidj A, Pijaudier-Cabot G. Coupling between progressive damage and permeability of concrete: analysis with a discrete model. International Journal for Numerical and Analytical Methods in Geomechanics, 2005; 29: 1005-1018.

[10] Nguyen V. H, Nedjar B, Torrenti J. M. Chemo-mechanical coupling behaviour of leached concrete Part II: Modelling. Nuclear Engineering and Design, 2007; 237: 2090-2097.

[11] Stora E, Bary B, He QC, Deville E, Montarnal P. Modelling and simulations of the chemo-mechanical behaviour of leached cement-based materials: Leaching process and induced loss of stiffness. Cement and Concrete Research, 2009; 39: 763-772.

[12] Luquot L, Gouze Ph. Experimental determination of porosity and permeability changes induced by massive injection of CO2 into carbonate reservoirs. Chemical Geology, 2009; 265: 148-159.

[13] Gouze P, Luquot L. X-ray microtomography characterization of porosity, permeability and reactive surface changes during dissolution. Journal of Contaminant Hydrology, 2011; 120-121: 45-55.

[14] Egermann P, Bekri S, Vizika O. An integrated approach to assess the petrophysical properties of rocks altered by rock/fluid interactions $\left(\mathrm{CO}_{2}\right.$ injection). Petrophysics 2010; 51: 32-40.

[15] Egermann P, Bemer E, Ziszner B. An experimental investigation of the rock properties evolution associated to different levels of $\mathrm{CO}_{2}$ injection like alternation processes. Paper SCA Presented at the Society of Core Analysts Symposium, Trondheim, Norway, 12-16 September 2006; SCA 2006-34.

[16] Izgec O, Demiral $\mathrm{B}$, Bertin $\mathrm{H}$, Akin $\mathrm{S} . \mathrm{CO}_{2}$ injection into saline carbonate aquifer formations I: laboratory investigations. Transport in Porous Media, 2008; 72: $1-24$.

[17] Xie S. Y, Shao J. F, Xu W. Y. Influence of chemical degradation on mechanical behaviour of a limestone. International Journal of Rock Mechnics 65 Minig Sciences, 2011; 48: 741-747.

[18] Canal J, Delgado J, Falcón I, Yang Q, Juncosa R, Barrientos V. Injection of $\mathrm{CO}_{2}$-saturated water through a siliceous sandstone plug from the Hontomin test 
site (Spain): experiment and modeling . Environmental Science 85 Technology, 2013; 47: 159-167.

[19] Qajar J, Francois N, Arns C. Micro-Tomographic characterization of dissolution-induced local porosity changes including fines migration in carbonate rock. SPE EOR Conference at Oil and Gas West Asia - EOR: Bulidings Towards Sustainable Growth 2012, Conference Organising Committee, Muscat, Oman, 2012; 117-134.

[20] Bemer J, Lombard J. M. From injectivity to integrity studies of $\mathrm{CO}_{2}$ geological storage. Chemical alteration effects on carbonates petrophysical and geomechanical properties. Oil \& Gas Science and Technology, 2009; 65: 445-459.

[21] Nguyen M. T, Bemer J, Dormieux L. Micromechanical modeling of carbonate geomechanical properties evolution during acid gas injection. Paper ARMA Presented at the 45th U.S. Mechanics/Geomechanics Symposium, San Francisco, USA, 26-29 June 2011; ARMA-11-207.

[22] Bachaud P, Berne P, Renard F, Sardin M, Leclerc J. P. Use of tracers to characterize the effects of a $\mathrm{CO}_{2}$-saturated brine on petrophysical properties of a low permeability carbonate caprock \& Minig SciencesChemical Engineering Research and Design, 2011; 89: 1817-1826.

[23] Peter M. A. Homogenisation in domains with evolving microstructure. Comptes Rendus Mécanique, 2007; 335: 357-362.

[24] Peter M. A. Homogenisation of a chemical degradation mechanism inducing an evolving microstructure. Comptes Rendus Mécanique, 2007; 335: 679-684.

[25] Peter M. A, Böhm M. Multiscale modelling of chemical degradation mechanism in prorus media with evolving microstructure. Multiscale Modelling 83 Simulations, 2009; 7: 1643-1668.

[26] Lewandowska J. Geomechanics in CO2 storage facilities (Pijaudier-Cabot G, Pereira J-M, eds). Wiley: Hoboken, 2008.

[27] Nguyen MT. Caractérisation géomechanique de la dégradation des roches sous l'effet de l'injection de gaz acides. PhD thesis, Université Paris-Est, France, 2012.

[28] Coussy B. Poromechanics, 2004; John Wiley and Sons Ltd. Chichester. 
[29] Doughty C, Preuss K. Modeling supercritical carbon dioxide injection in heterogeneous porous media. Vadose Zone Journal, 2004; 3: 837-847.

[30] Kumar A, Ozah R, Noh M, Pope G. A. Reservoir simulations of $\mathrm{CO}_{2}$ geological storage in deep saline aquifers. SPE Journal, 2005; 10: 336-348.

[31] Hovorka S. D, Benson S. M, Doughty C. Measuring permanence of $\mathrm{CO}_{2}$ storage in saline formations: The Frio experiment. Environmental Geoscience, 2006; 13: 105-121.

[32] Sarpialli P, McGrail P. Semi analytical approaches to modeling deep well injection of $\mathrm{CO}_{2}$ for geological sequestration. Energy Conversion and Management, 2002; 43, 185-198

[33] Nordbotten M. J, Celia A. M, Bachu S. Injection and storage of $\mathrm{CO}_{2}$ in deep saline aquifers: analytical solution for $\mathrm{CO}_{2}$ plume evolution during injection. Transport in Porous Media, 2005; 58: 339-360

[34] Sanchez-Palencia E. Non-Homogeneous Media and Vibration Theory. SpringerVerlag: Berlin, 1980.

[35] Auriault J-L. Heterogeneous medium. Is an equivalent macroscopic description possible? International Journal of Engineering Science, 1991; 29(7): 785-795.

[36] Auriault J-L, Sanchez-Palencia E. Etude du comportement macroscopique d'un milieu poreux saturé deformable. Journal de Mécanique,1977; 16: 575-603

[37] Françoise L. Contribution a l'etude des materiaux composites et de leur endommagement. PhD thesis, Université Pierre et Marie Curie, France, 1984.

[38] Bornet M, Bretheau T, Gilormini P. Homogénéisation en mécanique des matériaux 1: matériaux aléatoires élastiques et milieux périodiques. 2001; Hermes Science: Paris

[39] Gouze P, Melean Y, Le Borgne T, Dentz M, Carrera J. Non-Fickian dispersion in porous media explained by heterogeneous microscale matrix diffusion. Water Resources Research, 2008; 44: W11416.

[40] Boresi A. P, Schmidt R. J, Sidebottom O. M. Advanced Mechanics of Materials. 1993; Wiley: New York 
[41] Auriault J-L, Lewandowska J. Homogenization analysis of diffusion and adsorption macrotransport in porous media: macrotransport in the absence of advection. Géotechnique, 1993; 43(3): 457-469.

[42] Auriault J-L, Lewandowska J. Effective diffusion coefficient: from homogenization to experiment. Transport in Porous Media, 1997; 27: 205-223.

[43] Auriault J-L, Lewandowska J. Upscaling: cell symmetries and scale separation. Transport in Porous Media, 2001; 43: 473-485.

[44] Bear J. Dynamics of fluids in porous media. Elsvier: New York, 1972.

[45] Narsilio A. G, Buzzi O, Fityus S, Sup Yun T, Smith W. D. Upscaling of NavierStokes equations in porous media: Theoretical, numerical and experimental approach Computers and Geotechnics, 2009; 36: 1200-1206. 